Research Article

\title{
On Generalized Strongly Convex Functions and Unified Integral Operators
}

\author{
Timing Yu ${ }^{1},{ }^{1}$ Ghulam Farid ${ }^{D},{ }^{2}$ Kahkashan Mahreen, ${ }^{2}$ Chahn Yong Jung $\mathbb{D}^{3}$, \\ and Soo Hak Shim $\mathbb{D}^{4}$ \\ ${ }^{1}$ School of Economic Information Engineering, Southwestern University of Finance and Economics, Chengdu 611130, China
${ }^{2}$ Department of Mathematics, COMSATS University Islamabad, Attock Campus, Attock, Pakistan
${ }^{3}$ Department of Business Administration, Gyeongsang National University, Jinju 52828, Republic of Korea
${ }^{4}$ Department of Refrigeration and Air Conditioning Engineering, Chonnam National University, Yeosu 59626, Republic of Korea
}

Correspondence should be addressed to Timing Yu; yutiming@smail.swufe.edu.cn and Chahn Yong Jung; bb5734@gnu.ac.kr

Received 25 December 2020; Revised 2 February 2021; Accepted 5 August 2021; Published 30 August 2021

Academic Editor: Ghulam Mustafa

Copyright ( $\odot 2021$ Timing Yu et al. This is an open access article distributed under the Creative Commons Attribution License, which permits unrestricted use, distribution, and reproduction in any medium, provided the original work is properly cited.

In this paper, we define a strongly exponentially $(\alpha, h-m)$-convex function that generates several kinds of strongly convex and convex functions. The left and right unified integral operators of these functions satisfy some integral inequalities which are directly related to many unified and fractional integral inequalities. From the results of this paper, one can obtain various fractional integral operator inequalities that already exist in the literature.

\section{Introduction}

Mathematical inequalities are very important and useful in the study of various kinds of problems in mathematics, operation research, economics, physics, and engineering and in many other subjects of diverse fields. Integral inequalities provide support in the establishment of solutions to differential equations and solutions of boundary/initial value problems and their uniqueness. In recent years, mathematical inequalities for different kinds of fractional integrals are in the focus of researchers, and a lot of papers have been published on this subject. For instance, the classical inequalities such as the Chebyshev inequality, Minkowski inequality, Hadamard inequality, and Ostrowski inequality have been published for different kinds of fractional derivative/integral operators.

The existence of convex functions' revolutionary enhances the theory of mathematical inequalities. The properties and presentations of convex functions in different forms (geometric and analytical) create the room to invent other kinds of notions and functions. There are many types of convex functions that have been formulated from the analytical presentation of convex functions such as logarithmic convex, geometric convex, superquadratic, strongly convex, $h$-convex, $m$-convex, $\quad(\alpha, m)$-convex, $\quad(h, m)$-convex, $\quad$ and $(\alpha, h-m)$-convex functions. For different kinds of convex functions and their utilizations, one can see [1-6] and references therein. For applications in fractional integral inequalities, we refer the readers to [7-23] and references therein.

The aim of this paper is to study different kinds of convexities and fractional integral operators simultaneously in the form of unified integral inequalities. We will define a new function called strongly exponentially $(\alpha, h-m)$-convex function that unifies several kinds of convex functions in a single inequality (20). Also, left- and right-sided unified integral operators given in Definition 2 are utilized for this new class of convex functions to obtain the required results.

A huge amount of recent work in the theory of inequalities for real functions consists of the fractional integral inequalities and their applications. The Hadamard inequality is the most popular among classical inequalities which is studied extensively by researchers in near past decades for fractional integral operators. We will find an integral inequality of Hadamard type by applying operators (1) and (2) 
of the newly defined function. This will behave as a generalization as well as refinement of a lot of Hadamard and fractional Hadamard inequalities for convex functions of almost all kinds. Also, we will provide the upper bounds and bounds of several kinds of already known fractional integral operators connected with unified integral operators (1) and (2). Ultimately, the results of this paper work for all kinds of convexities and fractional integral operators that occur in the literature. Next, we give the results from the literature for fractional integral operators. A generalized fractional integral operator containing an extended generalized MittagLeffler function is defined as follows.

Definition 1 (see [7]). Let $\omega, \mu, \alpha, l, \gamma, c \in C$, $\mathfrak{R}(\mu), \mathfrak{R}(\alpha), \mathfrak{R}(l)>0$, and $\mathfrak{R}(c)>\mathfrak{R}(\gamma)>0$ with $p \geq 0$, $\delta>0$, and $0<k \leq \delta+\Re(\mu)$. Let $f \in L_{1}[a, b]$ and $x \in[a, b]$. Then, the generalized fractional integral operators $\varepsilon_{\mu, \alpha, l, \omega, a^{+}}^{\gamma, \delta, k, c} f$ and $\varepsilon_{\mu, \alpha, l, \omega, b^{-}}^{\gamma, \delta, k, c} f$ are defined by

$$
\begin{aligned}
& \left(\varepsilon_{\mu, \alpha, l, \omega, a^{+}}^{\gamma, \delta, k, c} f\right)(x, \omega ; p)=\int_{a}^{x}(x-t)^{\alpha-1} E_{\mu, \alpha, l}^{\gamma, \delta, k, c}\left(\omega(x-t)^{\mu} ; p\right) f(t) \mathrm{d} t, \\
& \left(\varepsilon_{\mu, \alpha, l, \omega, b^{-}}^{\gamma, \delta, k, c} f\right)(x, \omega ; p)=\int_{x}^{b}(t-x)^{\alpha-1} E_{\mu, \alpha, l}^{\gamma, \delta, k, c}\left(\omega(t-x)^{\mu} ; p\right) f(t) \mathrm{d} t,
\end{aligned}
$$

where

$$
E_{\mu, \alpha, l}^{\gamma, \delta, k, c}(t ; p)=\sum_{n=0}^{\infty} \frac{\beta_{p}(\gamma+n k, c-\gamma)}{\beta(\gamma, c-\gamma)} \frac{(c)_{n k}}{\Gamma(\mu n+\alpha)} \frac{t^{n}}{(l)_{n \delta}},
$$

is the extended generalized Mittag-Leffler function.

Mittag-Leffler function, introduced in 1903, is a generalization of the exponential function just by a single parameter with a convergence condition. Further generalization by another parameter was given by Wiman and further extended by Prabhakar and then by other authors; to see its importance, we suggest the reader to $[24,25]$. Next, we give recently established results in [26] for the fractional integral operators containing the extended generalized Mittag-Leffler function defined in [7] by utilizing $(\alpha, h-m)$-convex functions.

Theorem 1. Let $f:[a, b] \longrightarrow \mathbb{R}$ be a real-valued function. If $f$ is positive and $(\alpha, h-m)$-convex, $(\alpha, m) \in[0,1]^{2}, m \neq 0$, then for $\alpha^{\prime}, \beta \geq 1$, the following fractional integral inequality for generalized fractional integral operators holds:

$$
\begin{aligned}
& \left(\varepsilon_{\mu, \alpha^{\prime}, l, \omega, a^{+}}^{\gamma, \delta, k, c} f\right)(x ; p)+\left(\varepsilon_{\mu, \beta, l, \omega, b^{-}}^{\gamma, \delta, k, c} f\right)(x ; p) \\
& \leq(x-a) J_{\alpha^{\prime}-1, a^{+}}(x ; p)\left(f(a) \int_{0}^{1} h\left(z^{\alpha}\right) \mathrm{d} z+m f\left(\frac{x}{m}\right) \int_{0}^{1} h\left(1-z^{\alpha}\right) \mathrm{d} z\right) \\
& +(b-x) J_{\beta-1, b^{-}}(x ; p)\left(f(b) \int_{0}^{1} h\left(z^{\alpha}\right) \mathrm{d} z+m f\left(\frac{x}{m}\right) \int_{0}^{1} h\left(1-z^{\alpha}\right) \mathrm{d} z\right) .
\end{aligned}
$$

Theorem 2. Let $f:[a, b] \longrightarrow \mathbb{R}$ be a real-valued function. If $f$ is differentiable and $\left|f^{\prime}\right|$ is $(\alpha, h-m)$-convex, $(\alpha, m) \in[0,1]^{2}, \quad m \neq 0$, then for $\alpha^{\prime}, \beta \geq 1$, the following fractional integral inequality for generalized fractional integral operators holds:

$$
\begin{aligned}
& \mid\left(\varepsilon_{\mu, \alpha^{\prime}+1, l, \omega, a^{+}}^{\gamma, \delta, k, c} f\right)(x ; p)+\left(\varepsilon_{\mu, \beta+1, l, \omega, b^{-}}^{\gamma, \delta, k, c} f\right)(x ; p) \\
& \quad-\left(J_{\alpha^{\prime}-1, a^{+}}(x ; p) f(a)+J_{\beta-1, b^{-}}(x ; p) f(b)\right) \mid \\
& \leq(x-a) J_{\alpha^{\prime}-1, a^{+}}(x ; p)\left(\left|f^{\prime}(a)\right| \int_{0}^{1} h\left(z^{\alpha}\right) \mathrm{d} z+m\left|f^{\prime}\left(\frac{x}{m}\right)\right| \int_{0}^{1} h\left(1-z^{\alpha}\right) \mathrm{d} z\right) \\
& \quad+(b-x) J_{\beta-1, b^{-}}(x ; p)\left(\left|f^{\prime}(b)\right| \int_{0}^{1} h\left(z^{\alpha}\right) \mathrm{d} z+m\left|f^{\prime}\left(\frac{x}{m}\right)\right| \int_{0}^{1} h\left(1-z^{\alpha}\right) \mathrm{d} z\right) .
\end{aligned}
$$


Theorem 3. Let $f:[a, b] \longrightarrow \mathbb{R}, a>b$, be a real-valued function. If $f$ is positive and $(\alpha, h-m)$-convex, $(\alpha, m) \in[0,1]^{2}, \quad m \neq 0$, and $f((a+m b-x) / m)=f(x)$, then for $\alpha^{\prime}, \beta \geq 1$, the following fractional integral inequality holds:

$$
\begin{aligned}
& \frac{1}{h}\left(\frac{1}{2^{\alpha}}\right)+m h\left(1-\frac{1}{2^{\alpha}}\right) f\left(\frac{a+m b}{2}\right)\left[J_{\beta+1, b^{-}}(a ; p)+J_{\alpha^{\prime}+1, a^{+}}(b ; p)\right] \\
& \leq\left(\varepsilon_{\mu, \beta+1, l, \omega, b^{-}}^{\gamma, \delta, k, c}\right)(a ; p)+\left(\varepsilon_{\mu, \alpha^{\prime}+1, l, \omega, a^{+}}^{\gamma, \delta, k,} f\right)(b ; p) \\
& \leq\left[J_{\beta-1, b^{-}}(a ; p)+J_{\alpha^{\prime}-1, a^{+}}(b ; p)\right](b-a)^{2} \\
& \quad \times\left(f(b) \int_{0}^{1} h\left(z^{\alpha}\right) \mathrm{d} z+m f\left(\frac{a}{m}\right) \int_{0}^{1} h\left(1-z^{\alpha}\right) \mathrm{d} z\right) .
\end{aligned}
$$

Next, we give the definition of the unified integral operator based on a kernel which depends on a strictly increasing function along with two variables.

Definition 2 (see [16]). Let $f, g:[a, b] \longrightarrow \mathbb{R}, 0<a<b$, be the functions such that $f$ is positive and $f \in L_{1}[a, b]$ and $g$ is differentiable and strictly increasing. Also, let $(\phi / x)$ be an increasing function on $[a, \infty)$ and $\alpha, l, \gamma, c \in \mathbb{C}, p, \mu, \delta \geq 0$, and $0<k \leq \delta+\mu$. Then, for $x \in[a, b]$, the left and right integral operators are defined by

$$
\left({ }_{g} F_{\mu, \alpha, l, a^{+}}^{\phi, \gamma, \delta, c} f\right)(x, \omega ; p)=\int_{a}^{x} K_{x}^{y}\left(E_{\mu, \alpha^{\prime}, l}^{\gamma, \delta, k, c}, g ; \phi\right) f(y) d(g(y)),
$$

$$
\left({ }_{g} F_{\mu, \alpha, l, b^{-}}^{\phi, \gamma, \delta, k, c} f\right)(x, \omega ; p)=\int_{x}^{b} K_{y}^{x}\left(E_{\mu, \alpha^{\prime}, l}^{\gamma, \delta, c}, g ; \phi\right) f(y) d(g(y)),
$$

where the involved kernel is defined by

$$
K_{x}^{y}\left(E_{\mu, \alpha^{\prime}, l}^{\gamma, \delta, k,}, g ; \phi\right)=\frac{\phi(g(x)-g(y))}{g(x)-g(y)} E_{\mu, \alpha^{\prime}, l}^{\gamma, \delta, k}\left(\omega(g(x)-g(y))^{\mu} ; p\right) .
$$

The following property of the kernel given in (9) is used frequently in the whole paper.

P: let $g$ and $(\phi / I)$ be increasing functions. Then, for $m<t<n, m, n \in[a, b]$, the kernel $K_{m}^{n}\left(E_{\mu, \alpha^{\prime}, l}^{\gamma, \delta, k,}, g ; \phi\right)$ satisfies the following inequality:

$$
K_{t}^{m}\left(E_{\mu, \alpha^{\prime}, l}^{\gamma, \delta, k, c}, g ; \phi\right) g^{\prime}(t) \leq K_{n}^{m}\left(E_{\mu, \alpha^{\prime}, l}^{\gamma, \delta, k, c}, g ; \phi\right) g^{\prime}(t) .
$$

The reverse of inequality (10) holds when $g$ and $(\phi / I)$ are of opposite monotonicity. For further properties, see [27].

For suitable settings of functions $\phi$ and $g$ and certain values of parameters included in the Mittag-Leffler function, it unifies many fractional integral operators studied in $[10,18,20,25,28-37]$; see [38], Remarks 6 and 7. The following results are proved for unified integral operators of strongly convex functions in [39].

Theorem 4. Let $f:[a, b] \longrightarrow \mathbb{R}$ be a positive, integrable, and strongly convex function with $m \in(0,1]$. Let $g:[a, b] \longrightarrow \mathbb{R}$ be a differentiable and strictly increasing function; also, let $(\phi / x)$ be an increasing function on $[a, b]$. If $\alpha, \beta, l, \gamma, c \in \mathbb{R}_{+}, \quad p, \mu, \delta \geq 0$, and $0<k \leq \delta+\mu$, then for $x \in(a, b)$, we have

$$
\begin{aligned}
& \left({ }_{g} F_{\mu, \alpha, l, a^{+}}^{\phi, \gamma, \delta, k, c} f\right)(x, \omega ; p)+\left({ }_{g} F_{\mu, \beta, l, b^{-}}^{\phi, \gamma, \delta, k, c} f\right)(x, \omega ; p) \\
& \leq K_{x}^{a}\left(E_{\mu, \alpha, l}^{\gamma, \delta, k, c}, g ; \phi\right)((g(x)-g(a))(f(x)+f(a)) \\
& \left.\quad-\lambda(x-a)\left(2 I\left(a, x, I_{d} g\right)-(a+x) I(a, x, g)\right)\right) \\
& \quad+K_{b}^{x}\left(E_{\mu, \beta, l}^{\gamma, \delta, k, c}, g ; \phi\right)((g(b)-g(x))(f(b)+f(x)) \\
& \left.-\lambda(b-x)\left(2 I\left(x, b, I_{d} g\right)-(x+b) I(x, b, g)\right)\right) .
\end{aligned}
$$

Theorem 5. With the assumptions of Theorem 4, in addition, if $f(x)=f(a+b-x)$, then we have 


$$
\begin{aligned}
& f\left(\frac{a+b}{2}\right)\left({ }_{g} F_{\mu, \alpha, l, b^{-}}^{\phi, \gamma, \delta, k, c} 1\right)(a, \omega ; p)+\frac{\lambda}{4}\left({ }_{g} F_{\mu, \alpha, l, b^{-}}^{\phi, \gamma, \delta, k, c}(a+b-2 x)^{2}\right)(a, \omega ; p) \\
& \quad+f\left(\frac{a+b}{2}\right)\left({ }_{g} F_{\mu, \beta, l, a^{+}}^{\phi, \gamma, \delta, k, c} 1\right)(b, \omega ; p)+\frac{\lambda}{4}\left({ }_{g} F_{\mu, \beta, l, a^{+}}^{\phi, \gamma, \delta, k, c}(a+b-2 x)^{2}\right)(b, \omega ; p) \\
& \leq\left({ }_{g} F_{\mu, \alpha, l, b^{-}}^{\phi, \gamma, \delta, k, c} f\right)(a, \omega ; p)+\left({ }_{g} F_{\mu, \beta, l, a^{+}}^{\phi, \gamma, \delta, k, c} f\right)(b, \omega ; p) \\
& \leq\left(K_{b}^{a}\left(E_{\mu, \alpha, l}^{\gamma, \delta, k, c}, g ; \phi\right)+K_{b}^{a}\left(E_{\mu, \beta, l}^{\gamma, \delta, k, c}, g ; \phi\right)\right) \\
& \left((g(b)-g(a))(f(b)+f(a))-(b-a) \lambda\left(2 I\left(a, b, I_{d} g\right)-(a+b) I(a, b, g)\right)\right) .
\end{aligned}
$$

Theorem 6. Along with the assumptions of Theorem 4, if the strong convexity of $f$ is replaced with the strong convexity of $\left|f^{\prime}\right|$, then for $x \in(a, b)$, we have

$$
\begin{aligned}
& \left|\left(g_{\mu, \alpha, l, a^{+}}^{\phi, \gamma, \delta, k, c} f * g\right)(x, \omega ; p)+\left(g_{\mu, \beta, l, b^{-}}^{\phi, \gamma, \delta, k, c} f * g\right)(x, \omega ; p)\right| \leq K_{x}^{a}\left(E_{\mu, \alpha, l}^{\gamma, \delta, k, c}, g ; \phi\right) \\
& \quad \times\left((g(x)-g(a))\left(\left|f^{\prime}(x)\right|+\left|f^{\prime}(a)\right|\right)-\lambda(x-a)\left(2 I\left(a, x, I_{d} g\right)-(a+x) I(a, x, g)\right)\right) \\
& \quad+K_{b}^{x}\left(E_{\mu, \beta, l}^{\gamma, \delta, k, c}, g ; \phi\right)\left((g(b)-g(x))\left(\left|f^{\prime}(b)\right|+\left|f^{\prime}(x)\right|\right)\right. \\
& \left.\quad-\lambda(b-x)\left(2 I\left(x, b, I_{d} g\right)-(x+b) I(x, b, g)\right)\right),
\end{aligned}
$$

where $\left({ }_{g} F_{\mu, \alpha^{\prime}, l, a^{+}}^{\phi, \gamma, \delta, c} f * g\right)(x, \omega ; p)$ and $\left({ }_{g} F_{\mu, \beta, l, b^{-}}^{\phi, \gamma, \delta, c} f * g\right)(x, \omega ;$ $p)$ are the same as defined in Theorem 9.

The unified integral operators are also analyzed for $(\alpha, m)$-convex, $(h-m)$-convex, $(s, m)$-convex, and $(\alpha, h-m)$-convex functions in $[14,27,38,40]$. Here, we will unify bounds of unified and several kinds of fractional integral operators for different classes of convex, exponentially convex, strongly convex, and strongly exponentially convex functions in the results of this paper. Next, we give the definition of the $(h-m)$-convex function as follows.

Definition 3 (see [4]). Let $J \subseteq \mathbb{R}$ be an interval containing $(0,1)$, and let $h: J \longrightarrow \mathbb{R}$ be a nonnegative function. A function $f:[0, b] \longrightarrow \mathbb{R}$ is called an $(h-m)$-convex function if $f$ is nonnegative and for all $x, y \in[0, b]$, $m \in[0,1]$, and $t \in(0,1)$, one has

$$
f(t x+m(1-t) y) \leq h(t) f(x)+m h(1-t) f(y) .
$$

By suitably defining the function $h$ and giving particular values to parameter $m$, the above definition produces many known functions.

\section{Remark 1}

(i) If $m=1$, then the $h$-convex function can be obtained

(ii) If $h(t)=t$, then the $m$-convex function can be obtained

(iii) If $h(t)=t$ and $m=1$, then the convex function can be obtained

(iv) If $h(t)=1$ and $m=1$, the $p$-function can be obtained

(v) If $h(t)=t^{s}$ and $m=1$, then the $s$-convex function can be obtained

(vi) If $h(t)=(1 / t)$ and $m=1$, then the Godunova-Levin function can be obtained

(vii) If $h(t)=\left(1 / t^{s}\right)$ and $m=1$, then the $s$-Godunova-Levin function of the second kind can be obtained

The notion of the $(\alpha, m)$-convex function is defined as follows.

Definition 4 (see [3]). A function $f:[0, b] \longrightarrow \mathbb{R}, b>0$, is said to be $(\alpha, m)$-convex, where $(\alpha, m) \in[0,1]^{2}$, if 


$$
f(t x+m(1-t) y) \leq t^{\alpha} f(x)+m\left(1-t^{\alpha}\right) f(y)
$$

holds for all $x, y \in[0, b]$ and $t \in[0,1]$.

\section{Remark 2}

(i) If we consider $(\alpha, m)=(1, m)$, then (15) gives the definition of the $m$-convex function

(ii) If we consider $(\alpha, m)=(1,1)$, then (15) gives the definition of the convex function

(iii) If we consider $(\alpha, m)=(1,0)$, then (15) gives the definition of the star-shaped function

The notion of the $(\alpha, h-m)$-convex function is defined as follows.

Definition 5 (see [19]). Let $J \subseteq \mathbb{R}$ be an interval containing $(0,1)$, and let $h: J \longrightarrow \mathbb{R}$ be a nonnegative function. A function $f:[0, b] \longrightarrow \mathbb{R}$ is called an $(\alpha, h-m)$-convex function if $f$ is nonnegative and for all $x, y \in[0, b], t \in(0,1)$, and $(\alpha, m) \in[0,1]^{2}$, one has

$$
f(t x+m(1-t) y) \leq h\left(t^{\alpha}\right) f(x)+m h\left(1-t^{\alpha}\right) f(y) .
$$

Definition 6 (see [5]). Let $I$ be a nonempty convex subset of the normed space $(\mathbf{X},\|\|$.$) . A real-valued function f$ is said to be strongly convex, with modulus $\lambda \geq 0$, on $I$ if for each $x, y \in I$ and $t \in[0,1]$, we have

$$
f(t x+(1-t) y) \leq t f(x)+(1-t) f(y)-\lambda t(1-t)\|b-a\|^{2} .
$$

Definition 7 (see [41]). A function $f:[0, b] \longrightarrow \mathbb{R}$ is said to be an $(s, m)$-convex function, where $(s, m) \in[0,1]^{2}$ and $b>0$, if for every $x, y \in[0, b]$ and $t \in[0,1]$, we have

$$
f(t x+m(1-t) y) \leq t^{s} f(x)+m(1-t)^{s} f(y) .
$$

The notion of the strongly $(s, m)$-convex function is defined as follows.

Definition 8 (see [42]). A function $f:[0,+\infty) \longrightarrow \mathbb{R}$ is said to be a strongly $(s, m)$-convex function, with modulus $\lambda \geq 0$, for $(s, m) \in[0,1]^{2}$ if

$$
f(t a+m(1-t) b) \leq t^{s} f(a)+m(1-t)^{s} f(b)-\lambda m t(1-t)|b-a|^{2}
$$

holds for all $a, b \in[0,+\infty)$ and $t \in[0,1]$.

The rest of the paper is organized as follows: in Section 2, first, we define the strongly exponentially $(\alpha, h-m)$-convex function, and then upper bounds of unified integral operators of this function are obtained. Using the condition of symmetry and strongly exponentially $(\alpha, h-m)$-convexity of $\left|f^{\prime}\right|$, further bounds are obtained in the form of a modulus inequality. These results hold for all fractional integral operators comprised in [38], Remarks 6 and 7, deducible from unified integral operators (7) and (8) and for convex functions of various kinds.

\section{Main Results}

First, we give the definition of a new function called the strongly exponentially $(\alpha, h-m)$-convex function.

Definition 9. Let $J \subseteq \mathbb{R}$ be an interval containing $(0,1)$, and let $h: J \longrightarrow \mathbb{R}$ be a nonnegative function. A function $f:[0, b] \longrightarrow \mathbb{R}$ is called a strongly exponentially $(\alpha, h-m)$-convex function, with modulus $\lambda \geq 0$, if $f$ is nonnegative and for all $x, y \in[0, b], t \in(0,1), \eta \in \mathbb{R}$, and $(\alpha, m) \in[0,1]^{2}$, one has

$$
f(t x+m(1-t) y) \leq h\left(t^{\alpha}\right) \frac{f(x)}{e^{\eta x}}+m h\left(1-t^{\alpha}\right) \frac{f(y)}{e^{\eta y}}-\frac{m \lambda}{e^{(x+y) \eta}} h\left(t^{\alpha}\right) h\left(1-t^{\alpha}\right)|y-x|^{2} .
$$

The definition of the strongly $(\alpha, h-m)$-convex function is obtained as follows.

Definition 10. Let $J \subseteq \mathbb{R}$ be an interval containing $(0,1)$, and let $h: J \longrightarrow \mathbb{R}$ be a nonnegative function. A function $f:[0, b] \longrightarrow \mathbb{R}$ is called a strongly $(\alpha, h-m)$-convex function, with modulus $\lambda \geq 0$, if $f$ is nonnegative and for all $x, y \in[0, b], t \in(0,1)$, and $(\alpha, m) \in[0,1]^{2}$, one has

$$
f(t x+m(1-t) y) \leq h\left(t^{\alpha}\right) f(x)+m h\left(1-t^{\alpha}\right) f(y)-m \lambda h\left(t^{\alpha}\right) h\left(1-t^{\alpha}\right)|y-x|^{2}
$$

Many known and new convex, exponentially convex, strongly convex, and strongly exponentially convex functions can be obtained from the above definitions. The following theorem provides an upper bound for unified 
integral operators of strongly exponentially $(\alpha, h-m)$-convex functions.

Theorem 7. Let $f \in L_{1}[a, b], 0 \leq a<m b$, be a positive strongly exponentially $(\alpha, h-m)$-convex function, $m \in(0,1]$. Let $g$ be a differentiable and strictly increasing function; also, let $(\phi / x)$ be an increasing function and $h(x) h(y) \leq h(x+y)$. If $\alpha^{\prime}, \beta, l, \gamma, c \in \mathbb{R}_{+}, p, \mu \geq 0, \delta \geq 0$, and $0<k \leq \delta+\mu$, then for $x \in(a, b)$, we have

$$
\begin{aligned}
& \left(g_{\mu, \alpha^{\prime}, l, a^{+}}^{\phi, \gamma, \delta, k, c} f\right)(x, \omega ; p)+\left({ }_{g} F_{\mu, \beta, l, b^{-}}^{\phi, \gamma, \delta, k, c} f\right)(x, \omega ; p) \leq K_{x}^{a}\left(E_{\mu, \alpha^{\prime}, l}^{\gamma, \delta, k, c}, g ; \phi\right)(x-a) \\
& \left(\frac{f(a) X_{x}^{a}\left(z^{\alpha}, h ; g^{\prime}\right)}{e^{\eta a}}+\frac{m f(x / m) X_{x}^{a}\left(1-z^{\alpha}, h ; g^{\prime}\right)}{e^{\eta(x / m)}}-\frac{\lambda(x-m a)^{2} h(1)(g(x)-g(a))}{\left.m(x-a) e^{\eta(a+(x / m))}\right)}\right. \\
& +K_{b}^{x}\left(E_{\mu, \beta, l}^{\gamma, \delta, k, c}, g ; \phi\right)(b-x)\left(\frac{f(b) X_{x}^{b}\left(z^{\alpha}, h ; g^{\prime}\right)}{e^{\eta b}}+\frac{m f(x / m) X_{x}^{b}\left(1-z^{\alpha}, h ; g^{\prime}\right)}{e^{\eta(x / m)}}\right. \\
& \quad-\frac{\lambda(b m-x)^{2} h(1)(g(b)-g(x))}{\left.m(b-x) e^{\eta(b+(x / m))}\right)}
\end{aligned}
$$

where $X_{x}^{a}\left(z^{\alpha}, h ; g^{\prime}\right)=\int_{0}^{1} h\left(z^{\alpha}\right) g^{\prime}(x-z(x-a)) d z \quad$ and $X_{x}^{a}\left(1-z^{\alpha}, h ; g^{\prime}\right)=\int_{0}^{1} h\left(1-z^{\alpha}\right) g^{\prime}(x-z(x-a)) d z$.

Proof. By using (P), the following inequalities hold:

$$
\begin{aligned}
& K_{x}^{t}\left(E_{\mu, \alpha^{\prime}, l}^{\gamma, \delta, k, c}, g ; \phi\right) g^{\prime}(t) \leq K_{x}^{a}\left(E_{\mu, \alpha^{\prime}, l}^{\gamma, \delta, k, c}, g ; \phi\right) g^{\prime}(t), \quad a<t<x, \\
& K_{t}^{x}\left(E_{\mu, \alpha^{\prime}, l}^{\gamma, \delta, k, c}, g ; \phi\right) g(t) \leq K_{b}^{x}\left(E_{\mu, \alpha^{\prime}, l}^{\gamma, \delta, k, c}, g ; \phi\right) g^{\prime}(t), \quad x<t<b .
\end{aligned}
$$

Using strongly exponentially $(\alpha, h-m)$-convexity of $f$, we have

$$
\begin{aligned}
f(t) \leq & h\left(\frac{x-t}{x-a}\right)^{\alpha} \frac{f(a)}{e^{\eta a}}+m h\left(1-\left(\frac{x-t}{x-a}\right)^{\alpha}\right) \frac{f(x / m)}{e^{\eta(x / m)}} \\
& -\frac{\lambda(x-a m)^{2}}{m e^{\eta(a+(x / m))}} h\left(\frac{x-t}{x-a}\right)^{\alpha} h\left(1-\left(\frac{x-t}{x-a}\right)^{\alpha}\right), \\
f(t) \leq & h\left(\frac{t-x}{b-x}\right)^{\alpha} \frac{f(b)}{e^{\eta b}}+m h\left(1-\left(\frac{t-x}{b-x}\right)^{\alpha}\right) \frac{f(x / m)}{e^{\eta(x / m)}} \\
& -\frac{\lambda(b m-x)^{2}}{m e^{\eta(b+(x / m))}} h\left(\frac{t-x}{b-x}\right)^{\alpha} h\left(1-\left(\frac{t-x}{b-x}\right)^{\alpha}\right) .
\end{aligned}
$$

From (23) and (25), the following integral inequality holds true:

$$
\begin{aligned}
& \int_{a}^{x} K_{x}^{t}\left(E_{\mu, \alpha^{\prime}, l}^{\gamma, c, k}, g ; \phi\right) f(t) d(g(t)) \leq f(a) K_{x}^{a}\left(E_{\mu, \alpha^{\prime}, l}^{\gamma, \delta, k,}, g ; \phi\right) \int_{a}^{x} h\left(\frac{x-t}{x-a}\right)^{\alpha} d(g(t)) \\
& +m \frac{f(x / m)}{e^{\eta(x / m)}} K_{x}^{a}\left(E_{\mu, \alpha^{\prime}, l}^{\gamma, \delta, k, c}, g ; \phi\right) \int_{a}^{x} h\left(1-\left(\frac{x-t}{x-a}\right)^{\alpha}\right) d(g(t)) \\
& -\frac{\lambda(b m-x)^{2}}{m} K_{x}^{a}\left(E_{\mu, \alpha^{\prime}, l}^{\gamma, \delta, c, c}, g\right) \int_{a}^{x} h\left(1-\left(\frac{x-t}{x-a}\right)^{\alpha}\right) h\left(\frac{x-t}{x-a}\right)^{\alpha} d(g(t)) .
\end{aligned}
$$

By using (7) of Definition 2 on the left-hand side and by setting $z=(x-t) /(x-a)$ on the right-hand side, the following inequality is obtained: 


$$
\begin{aligned}
& \left({ }_{g} F_{\mu, \alpha^{\prime}, l, a^{+}}^{\phi, \gamma, \delta, k} f\right)(x, \omega ; p) \leq K_{x}^{a}\left(E_{\mu, \alpha^{\prime}, l}^{\gamma, \delta, k, c}, g ; \phi\right)(x-a) \\
& \quad \times\left(\frac{f(a)}{e^{\eta a}} \int_{0}^{1} h\left(z^{\alpha}\right) g^{\prime}(x-z(x-a)) \mathrm{d} z+\frac{m f(x / m)}{e^{\eta(x / m)}} \int_{0}^{1} h\left(1-z^{\alpha}\right) g^{\prime}(x-z(x-a)) \mathrm{d} z\right. \\
& \left.\quad-\frac{\lambda(x-a m)^{2}}{m e^{\eta(a+(x / m))}} \int_{0}^{1} h\left(1-z^{\alpha}\right) h\left(z^{\alpha}\right) g^{\prime}(x-z(x-a)) \mathrm{d} z\right) .
\end{aligned}
$$

The above inequality can be written as follows:

$$
\begin{aligned}
& \left({ }_{g} F_{\mu, \alpha^{\prime}, l, a^{+}}^{\phi, \gamma, \delta, c} f\right)(x, \omega ; p) \leq K_{x}^{a}\left(E_{\mu, \alpha^{\prime}, l}^{\gamma, \delta, k, c}, g ; \phi\right)(x-a) \\
& \quad \times\left(\frac{f(a)}{e^{\eta a}} X_{x}^{a}\left(z^{\alpha}, h ; g^{\prime}\right)+m f \frac{f(x / m)}{e^{\eta(x / m)}} X_{x}^{a}\left(1-z^{\alpha}, h ; g^{\prime}\right)-\frac{\lambda(x-m a)^{2} h(1)(g(x)-g(a))}{m(x-a) e^{\eta(a+(x / m))}}\right) .
\end{aligned}
$$

On the contrary, multiplying (24) and (26) and adopting the same pattern as we did for (23) and (25), the following inequality holds true:

$$
\begin{aligned}
& \left({ }_{g} F_{\mu, \beta, l, b^{-}}^{\phi, \gamma, k, k} f\right)(x, \omega ; p) \leq K_{b}^{x}\left(E_{\mu, \beta, l}^{\gamma, \delta, k, c}, g ; \phi\right)(b-x) \\
& \quad \times\left(\frac{f(b)}{e^{\eta b}} \int_{0}^{1} h\left(z^{\alpha}\right) g^{\prime}(x-z(x-b)) \mathrm{d} z+\frac{m f(x / m)}{e^{\eta(x / m)}} \int_{0}^{1} h\left(1-z^{\alpha}\right) g^{\prime}(x-z(x-b)) \mathrm{d} z\right. \\
& \left.\quad-\frac{\lambda(b m-x)^{2}}{m e^{\eta(b+(x / m))}} \int_{0}^{1} h\left(1-z^{\alpha}\right) h\left(z^{\alpha}\right) g^{\prime}(x-z(x-b)) \mathrm{d} z\right)
\end{aligned}
$$

The above inequality can be written as follows:

$$
\begin{aligned}
& \left({ }_{g} F_{\mu, \beta, l, b^{-}}^{\phi, \gamma, \delta, k, c} f\right)(x, \omega ; p) \leq K_{b}^{x}\left(E_{\mu, \beta, l}^{\gamma, \delta, k, c}, g ; \phi\right)(b-x) \\
& \quad \times\left(\frac{f(b)}{e^{\eta b}} X_{x}^{b}\left(z^{\alpha}, h ; g^{\prime}\right)+m \frac{f(x / m)}{e^{\eta(x / m)}} X_{x}^{b}\left(1-z^{\alpha}, h ; g^{\prime}\right)-\frac{\lambda(b m-x)^{2} h(1)(g(b)-g(x))}{m(b-x) e^{\eta(b+(x / m))}}\right) .
\end{aligned}
$$

By adding (29) and (31), (22) can be obtained.

Corollary 1. If we take $\eta=0$ and $m=\alpha=1$, then (22) holds for strongly h-convex functions.

$$
\begin{aligned}
& \left({ }_{g} F_{\mu, \alpha^{\prime}, l, a^{+}}^{\phi, \gamma, \delta, k, c} f\right)(x, \omega ; p)+\left({ }_{g} F_{\mu, \beta, l, b^{-}}^{\phi, \gamma, \delta, k, c} f\right)(x, \omega ; p) \leq K_{x}^{a}\left(E_{\mu, \alpha^{\prime}, l}^{\gamma, \delta, k, c}, g ; \phi\right)(x-a) \\
& \left(f(a) Y_{x}^{a}\left(z, h ; g^{\prime}\right)+f(x) Y_{x}^{a}\left(y, h ; g^{\prime}\right)-\lambda(x-a) h(1)(g(x)-g(a))\right) \\
& \quad+K_{b}^{x}\left(E_{\mu, \beta, l}^{\gamma, \delta, k, c}, g ; \phi\right)(b-x)\left(f(b) Y_{x}^{b}\left(z, h ; g^{\prime}\right)+f(x) Y_{x}^{b}\left(y, h ; g^{\prime}\right)\right. \\
& \quad-\lambda(b-x) h(1)(g(b)-g(x))) .
\end{aligned}
$$


The following remark summarizes the connection of Theorem 7 with already known results.

\section{Remark 3}

(i) If we consider $\eta=0$ and $\alpha=1$ in (22), then Theorem 1 of [40] can be obtained

(ii) If we consider $\eta=0, \alpha^{\prime}=\beta$, and $h(t)=t$ in (22), then Theorem 1 of [14] can be obtained

(iii) If we consider $\eta=0, \lambda=0, \alpha^{\prime}=\beta, m=\alpha=1$, and $h(t)=t$ in (22), then Theorem 8 of [38] can be obtained

(iv) If we consider $\quad \eta=0, \quad \lambda=0$, $\phi(t)=\left(\Gamma\left(\alpha^{\prime}\right) t^{\left(\alpha^{\prime} / k\right)} / k \Gamma_{k}\left(\alpha^{\prime}\right)\right), \quad h(t)=g(t)=t$, $\alpha=m=1$, and $p=\omega=0$ in (22), then Theorem 1 of [13] can be obtained

(v) If we consider $\eta=0, \lambda=0$, and $\alpha^{\prime}=\beta$ in the result of (iv), then Corollary 1 of [13] can be obtained

(vi) If we consider $\eta=0, \lambda=0, k=1$, and $x=a$ or $x=b$ in the result of (v), then Corollary 2 of [13] can be obtained

(vii) If we consider $\eta=0, \lambda=0, \quad k=1, \quad$ and $x=(a+b) / 2$ in the result of $(\mathrm{v})$, then Corollary 3 of [13] can be obtained

(viii) If we consider $\eta=0, \lambda=0, \phi(t)=t^{\alpha^{\prime}}, \alpha=1$, and $g(t)=t$ in (22), then Theorem 1 of [11] can be obtained

(ix) If we consider $\eta=0, \lambda=0, \phi(t)=t^{\alpha^{\prime}}, \alpha=m=1$, and $h(t)=g(t)=t$ in (22), then Corollary 1 of [11] can be obtained

(x) If we consider $\eta=0, \lambda=0, \phi(t)=\Gamma\left(\alpha^{\prime}\right) t^{\alpha^{\prime}}, \alpha=1$, $p=\omega=0$, and $g(t)=t$ in (22), then Theorem 1 of [12] can be obtained

(xi) If we consider $\eta=0, \lambda=0$, and $\alpha^{\prime}=\beta$ in the result of (x), then Corollary 1 of [12] can be obtained

(xii) If we consider $\eta=0, \quad \lambda=0, \quad \alpha^{\prime}=\beta$, $\phi(t)=\Gamma\left(\alpha^{\prime}\right) t^{\alpha^{\prime}}, p=\omega=0, g(t)=t$, and $\alpha=1$ and using Remark (2) of [12] in (22), then Corollary 2 of [12] can be obtained

(xiii) If we consider $\eta=0, \lambda=0, \phi(t)=\Gamma\left(\alpha^{\prime}\right) t^{\alpha^{\prime}}, \alpha=1$, $p=\omega=0, h(t)=1, h(t)=t^{p}$, respectively, and $g(t)=t$ in (22), then Theorem 3 of [12] can be obtained

(xiv) If we consider $\eta=0, \lambda=0, \phi(t)=\Gamma\left(\alpha^{\prime}\right) t^{\alpha^{\prime}}$, $p=\omega=0, m=\alpha=1$, and $g(t)=h(t)=t$ in (22), then Theorem 1 of [15] can be obtained

(xv) If we consider $\eta=0, \lambda=0$, and $\alpha^{\prime}=\beta$ in the result of (xiv), then Corollary 1 of [15] can be obtained

(xvi) If we consider $\eta=0, \lambda=0, \phi(t)=\Gamma\left(\alpha^{\prime}\right) t^{\alpha^{\prime}}$, $p=\omega=0, m=\alpha=1$, and $h(t)=t$ in (22), then Theorem 1 of [17] can be obtained (xvii) If we consider $\eta=0, \quad \lambda=0, \quad \alpha^{\prime}=\beta$, $\phi(t)=\Gamma\left(\alpha^{\prime}\right) t^{\alpha^{\prime}}, p=\omega=0, m=\alpha=1$, and $h(t)=$ $t$ in (22), then Corollary 1 of [17] can be obtained

(xviii) If we consider $\eta=0, \lambda=0, \phi(t)=t^{\alpha^{\prime}}, g(t)=t$, $h(t)=t^{s}$, and $\alpha=m=1$ in (22), then Theorem 2.1 of [43] can be obtained

(xix) If we consider $\eta=0, \lambda=0, \alpha^{\prime}=\beta, \phi(t)=t^{\alpha^{\prime}}$, $g(t)=t, m=\alpha=1$, and $h(t)=t^{s}$ in (22), then Corollary 2.1 of [43] can be obtained

(xx) If we consider $\eta=0, \lambda=0, p=\omega=0, \alpha=1$, and $h(t)=t^{s}$ in (22), then Theorem 1 of [44] can be obtained

(xxi) If we consider $\eta=0, \lambda=0, \phi(t)=t^{\alpha^{\prime}}, h(t)=t^{s}$, $\alpha=1$, and $g(t)=t$ in (22), then Theorem 1 of [41] can be obtained

(xxii) If we consider $\eta=0, \lambda=0$, and $\alpha^{\prime}=\beta$ in the result of (xxi), then Corollary 1 of [41] can be obtained

(xxiii) If we consider $\eta=0, \lambda=0, \phi(t)=\left(\Gamma\left(\alpha^{\prime}\right)\right.$ $\left.t^{\left(\alpha^{\prime} / k\right)} / k \Gamma_{k}\left(\alpha^{\prime}\right)\right), h(t)=z$, and $p=\omega=0$ in (22), then Theorem 1 of [21] can be obtained

(xxiv) If we consider $\eta=0, \lambda=0$, and $\alpha^{\prime}=\beta$ in the result of (xxiii), then Corollary 1 of [21] can be obtained

The following lemma will be used for the proof of the upcoming theorem.

Lemma 1. Let $f:[a, b] \longrightarrow \mathbb{R}, 0 \leq a<m b$, be a strongly exponentially $(\alpha, h-m)$-convex function. If $\left(f(x) / e^{x}\right)=\left(f((a+m b-x) / m) / e^{(a+m b-x) / m}\right), m \neq 0$, then the following inequality holds:

$$
\begin{aligned}
f\left(\frac{a+m b}{2}\right) \leq & \left(h\left(\frac{1}{2^{\alpha}}\right)+m h\left(\frac{2^{\alpha}-1}{2^{\alpha}}\right)\right) \frac{f(x)}{e^{\eta x}} \\
& -\frac{\lambda}{m} h\left(\frac{1}{2^{\alpha}}\right) h\left(\frac{2^{\alpha}-1}{2^{\alpha}}\right) \frac{(a+m b-x-m x)^{2}}{e^{\eta((a+m b+x(m-1)) / m)}} .
\end{aligned}
$$

Proof. As $f$ is a strongly exponentially $(\alpha, h-m)$-convex function, we have

$$
\begin{aligned}
f\left(\frac{a+m b}{2}\right) \leq & h\left(\frac{1}{2^{\alpha}}\right) \frac{f((1-t) a+m t b)}{e^{\eta((1-t) a+m t b)}} \\
& +m h\left(\frac{2^{\alpha}-1}{2^{\alpha}}\right) \frac{f(t a+m(1-t) b / m)}{e^{\eta((t a+m(1-t) b) / m)}} \\
& -\frac{\lambda}{m} h\left(\frac{1}{2^{\alpha}}\right) h\left(\frac{2^{\alpha}-1}{2^{\alpha}}\right) \\
& \frac{(t(1+m)(a-m b)+m b-m a)^{2}}{e^{\eta((1-t) a+m t b+((t a+m(1-t) b) / m))}} .
\end{aligned}
$$


Let $x=a(1-t)+m t b$. Then, we have $a+m b-x=t a+$ $m(1-t) b$, and using the given condition on $f$, inequality (33) can be obtained.

The following result provides bounds in the form of Hadamard inequality for strongly exponentially $(\alpha, h-m)$-convex functions.
Theorem 8. The conditions on $f, g, h$, and $\phi$ are the same as those in Theorem 7, and in addition, if $\left(f(x) / e^{x}\right)=\left(f((a+m b-x) / m) / e^{(a+m b-x) / m}\right)$, then we have

$$
\begin{gathered}
\frac{A(\eta)}{h\left(1 / 2^{\alpha}\right)+m h\left(\left(2^{\alpha}-1\right) / 2^{\alpha}\right)}\left(f\left(\frac{a+m b}{2}\right)\left(\left({ }_{g} F_{\mu, \alpha^{\prime}, l, a^{+}}^{\phi, \gamma, \delta, k, c} 1\right)(b, \omega ; p)+\left({ }_{g} F_{\mu, \beta, l, b^{-}}^{\phi, \gamma, \delta, k} 1\right)(a, \omega ; p)\right)\right. \\
+\frac{\lambda}{m} h\left(\frac{1}{2^{\alpha}}\right) h\left(\frac{2^{\alpha}-1}{2^{\alpha}}\right)\left(\frac{\left.{ }_{g} F_{\mu, \alpha^{\prime}, l, a^{+}}^{\phi, \gamma, \delta, c}(a+m b-x-m x)^{2}\right)(b, \omega ; p)}{e^{\eta((m b+a-b) / m)}}\right. \\
\left.+\frac{\left({ }_{g} F_{\mu, \beta, l, l, b^{-}}^{\phi, \gamma, k, c}(a+m b-x-m x)^{2}\right)(a, \omega ; p)}{e^{\eta(b)}}\right) \leq\left({ }_{g} F_{\mu, \alpha^{\prime}, l, a^{+}}^{\phi, \gamma, k} f\right)(b, \omega ; p) \\
+\left({ }_{g} F_{\mu, \beta, l, k, b^{-}}^{\phi, \gamma, \delta, k} f\right)(a, \omega ; p) \leq(b-a)\left(K_{b}^{a}\left(E_{\mu, \alpha^{\prime}, l}^{\gamma, \delta, k, c}, g ; \phi\right)+K_{b}^{a}\left(E_{\mu, \beta, l}^{\gamma, \delta, k, c}, g ; \phi\right)\right) \\
\left(\frac{f(b) X_{b}^{a}\left(z^{\alpha}, h ; g^{\prime}\right)}{e^{\eta b}}+\frac{m f(a / m) X_{b}^{a}\left(1-z^{\alpha}, h ; g^{\prime}\right)}{e^{\eta(a / m)}}-\frac{\lambda(a-b m)^{2} h(1)(g(b)-g(a))}{\left.m(b-a) e^{\eta(b+(a / m))}\right),}\right.
\end{gathered}
$$

where $A(\eta)=e^{\eta a}$ when $\eta \geq 0$ and $A(\eta)=e^{\eta b}$ when $\eta<0 . \quad$ Proof. By using $(\mathbf{P})$, the following inequalities hold:

$$
\begin{aligned}
& K_{x}^{a}\left(E_{\mu, \beta, l}^{\gamma, \delta, k, c}, g ; \phi\right) g^{\prime}(x) \leq K_{b}^{a}\left(E_{\mu, \beta, l}^{\gamma, \delta, k, c}, g ; \phi\right) g^{\prime}(x), \quad a<x<b, \\
& K_{b}^{x}\left(E_{\mu, \alpha^{\prime}, l}^{\gamma, \delta, k, c}, g ; \phi\right) g^{\prime}(x) \leq K_{b}^{a}\left(E_{\mu, \alpha^{\prime}, l}^{\gamma, \delta, k, c}, g ; \phi\right) g^{\prime}(x), \quad a<x<b .
\end{aligned}
$$

Using the strongly exponentially $(\alpha, h-m)$-convexity of $f$, we have

$$
\begin{aligned}
f(x) \leq & h\left(\frac{x-a}{b-a}\right)^{\alpha} \frac{f(b)}{e^{\eta} b}+m h\left(1-\left(\frac{x-a}{b-a}\right)^{\alpha}\right) \frac{f(a / m)}{e^{\eta(a / m)}} \\
& -\frac{\lambda(a-b m)^{2}}{m e^{\eta(b+(a / m))}} h\left(\frac{x-a}{b-a}\right)^{\alpha} h\left(1-\left(\frac{x-a}{b-a}\right)^{\alpha}\right) .
\end{aligned}
$$

Multiplying (36) and (38) and integrating the resulting inequality over $[a, b]$, we obtain

$$
\begin{aligned}
& \int_{a}^{b} K_{x}^{a}\left(E_{\mu, \beta, l}^{\gamma, \delta, c, c}, g ; \phi\right) f(x) d(g(x)) \leq \frac{f(b)}{e^{\eta b}} K_{b}^{a}\left(E_{\mu, \beta, l}^{\gamma, \delta, k, c}, g ; \phi\right) \\
& \quad \times \int_{a}^{b} h\left(\frac{x-a}{b-a}\right)^{\alpha} d(g(x))+m \frac{f(a / m)}{e^{\eta(a / m)}} K_{b}^{a} \\
& \left(E_{\mu, \beta, l}^{\gamma, \delta, k, c}, g ; \phi\right) \int_{a}^{b} h\left(1-\left(\frac{x-a}{b-a}\right)^{\alpha}\right) d(g(x)) \\
& -\frac{\lambda(a-b m)^{2}}{m e^{\eta(b+(a / m))}} \int_{a}^{b} h\left(\frac{x-a}{b-a}\right)^{\alpha} h\left(1-\left(\frac{x-a}{b-a}\right)^{\alpha}\right) d(g(x)) .
\end{aligned}
$$


Now, by using (7) of Definition 2 on the left-hand side and by setting $z=(x-a) /(b-a)$ on the right-hand side, the following inequality is obtained:

$$
\begin{aligned}
\left({ }_{g} F_{\mu, \beta, l, b^{-}}^{\phi, \gamma, \delta, k, c} f\right)(a, \omega ; p) \leq & K_{b}^{a}\left(E_{\mu, \beta, l}^{\gamma, \delta, k, c}, g ; \phi\right)(b-a) \\
& \times\left(\frac{f(b)}{e^{\eta b}} \int_{0}^{1} h\left(z^{\alpha}\right) g^{\prime}(a+z(b-a)) \mathrm{d} z+m \frac{f(a / m)}{e^{\eta(a / m)}} \int_{0}^{1} h\left(1-z^{\alpha}\right) g^{\prime}(a+z(b-a)) \mathrm{d} z\right. \\
& \left.-\frac{\lambda(a-b m)^{2}}{m e^{\eta(b+(a / m))}} \int_{0}^{1} h\left(z^{\alpha}\right) h\left(1-z^{\alpha}\right) g^{\prime}(a+z(b-a)) \mathrm{d} z\right) .
\end{aligned}
$$

The above inequality can be written as

$$
\begin{aligned}
\left(g_{g, \beta, l, b^{-}}^{\phi, \gamma, \delta, k, c} f\right)(a, \omega ; p) \leq & K_{b}^{a}\left(E_{\mu, \beta, l}^{\gamma, \delta, k, c}, g ; \phi\right)(b-a) \\
& \times\left(\frac{f(b)}{e^{\eta b}} X_{b}^{a}\left(\left(z^{\alpha}, h ; g^{\prime}\right)+m \frac{f(a / m)}{e^{\eta(a / m)}} X_{b}^{a}\left(1-z^{\alpha}, h ; g^{\prime}\right)-\frac{\lambda(a-b m)^{2} h(1)(g(b)-g(a))}{m(b-a) e^{\eta(b+(a / m))}}\right) .\right.
\end{aligned}
$$

Adopting the same pattern of simplification as we did for (36) and (38), the following inequality can be observed from

(37) and (38):

$$
\begin{aligned}
\left({ }_{g} F_{\mu, \alpha^{\prime}, l, a^{+}}^{\phi, \gamma, \delta, c} f\right)(b, \omega ; p) \leq & K_{b}^{a}\left(E_{\mu, \alpha^{\prime}, l}^{\gamma, \delta, k, c}, g ; \phi\right)(b-a) \\
& \times\left(\frac{f(b)}{e^{\eta b}} X_{b}^{a}\left(z^{\alpha}, h ; g^{\prime}\right)+m \frac{f(a / m)}{e^{\eta(a / m)}} X_{b}^{a}\left(1-z^{\alpha}, h ; g^{\prime}\right)-\frac{\lambda(a-b m)^{2} h(1)(g(b)-g(a))}{m(b-a) e^{\eta(b+(a / m))}}\right) .
\end{aligned}
$$

By adding (41) and (42), the following inequality can be obtained:

$$
\begin{aligned}
\left({ }_{g} F_{\mu, \alpha^{\prime}, l, a^{+}}^{\phi, \gamma} f\right)(b, \omega ; p)+\left({ }_{g} F_{\mu, \beta, l, b^{-}}^{\phi, \gamma, \delta, k, c} f\right)(a, \omega ; p) \leq & (b-a)\left(K_{b}^{a}\left(E_{\mu, \alpha^{\prime}, l}^{\gamma, \delta, k, c}, g ; \phi\right)+K_{b}^{a}\left(E_{\mu, \beta, l}^{\gamma, \delta, k, c}, g ; \phi\right)\right) \\
& \left(\frac{f(b)}{e^{\eta b}} X_{b}^{a}\left(z^{\alpha}, h ; g^{\prime}\right)+m \frac{f(a / m)}{e^{\eta(a / m)}} X_{b}^{a}\left(1-z^{\alpha}, h ; g^{\prime}\right)\right. \\
& -\frac{\lambda(a-b m)^{2} h(1)(g(b)-g(a))}{\left.m(b-a) e^{\eta(b+(a / m))}\right) .}
\end{aligned}
$$

Multiplying both sides of (33) by $K_{x}^{a}\left(E_{\mu, \beta, l}^{\gamma, \delta, k, c}, g\right.$; $\phi) d(g(x))$ and integrating over $[a, b]$, we have 


$$
\begin{aligned}
& f\left(\frac{a+m b}{2}\right) \int_{a}^{b} K_{x}^{a}\left(E_{\mu, \beta, l}^{\gamma, \delta, k, c}, g ; \phi\right) d(g(x)) \\
& \leq\left(h\left(\frac{1}{2^{\alpha}}\right)+m h\left(\frac{2^{\alpha}-1}{2^{\alpha}}\right)\right) \int_{a}^{b} K_{x}^{a}\left(E_{\mu, \beta, l}^{\gamma, \delta, k, c}, g ; \phi\right) \frac{f(x)}{e^{\eta x}} d(g(x)) \\
& \quad-\frac{\lambda}{m} h\left(\frac{1}{2^{\alpha}}\right) h\left(\frac{2^{\alpha}-1}{2^{\alpha}}\right) \int_{a}^{b} K_{x}^{a}\left(E_{\mu, \beta, l}^{\gamma, \delta, k, c}, g ; \phi\right) \frac{(a+m b-x-m x)^{2}}{e^{\eta((a+m b+x(m-1)) / m)}} d(g(x)) .
\end{aligned}
$$

From Definition 2, the following inequality is obtained:

$$
\begin{aligned}
& \frac{A(\eta)}{h\left(1 / 2^{\alpha}\right)+m h\left(\left(2^{\alpha}-1\right) / 2^{\alpha}\right)}\left(f\left(\frac{a+m b}{2}\right)\left({ }_{g} F_{\mu, \beta, l, b^{-}}^{\phi, \gamma, \delta, k, c} 1\right)(a, \omega ; p)+\frac{\lambda}{m e^{\eta(b)}} h\left(\frac{1}{2^{\alpha}}\right)\right. \\
& \left.\times h\left(\frac{2^{\alpha}-1}{2^{\alpha}}\right)\left({ }_{g_{\mu, \beta, l, b^{-}}} F^{\phi, \gamma, \delta, k, c}(a+m b-x-m x)^{2}\right)(a, \omega ; p)\right) \leq\left({ }_{g^{2}}{ }_{\mu, \beta, l, b^{-}}^{\phi, \gamma, \delta, k, c} f\right)(a, \omega ; p) .
\end{aligned}
$$

Similarly, multiplying both sides of (33) by $K_{b}^{x}\left(E_{\mu, \alpha^{\prime \prime}}^{\gamma, \delta, k,}, g ; \phi\right) d(g(x))$ and integrating over $[a, b]$, we have

$$
\begin{aligned}
& \frac{A(\eta)}{h\left(1 / 2^{\alpha}\right)+m h\left(\left(2^{\alpha}-1\right) / 2^{\alpha}\right)}\left(f\left(\frac{a+m b}{2}\right)\left({ }_{g} F_{\mu, \alpha^{\prime}, l, a^{+}}^{\phi, \gamma, \delta, k, c} 1\right)(b, \omega ; p)+\frac{\lambda}{m e^{\eta((m b+a-b) / m)}} h\left(\frac{1}{2^{\alpha}}\right)\right. \\
& \left.\times h\left(\frac{2^{\alpha}-1}{2^{\alpha}}\right)\left({ }_{g} F_{\mu, \alpha^{\prime}, l, a^{+}}^{\phi, \gamma, \delta, c}(a+m b-x-m x)^{2}\right)(b, \omega ; p)\right) \leq\left({ }_{g} F_{\mu, \alpha^{\prime}, l, a^{+}}^{\phi, \gamma, \delta, c} f\right)(b, \omega ; p) .
\end{aligned}
$$

From (43), (45), and (46), inequality (35) can be obtained.
Corollary 2. If we take $\eta=0$ and $m=\alpha=1$, then (35) holds for strongly h-convex functions.

$$
\begin{aligned}
& \frac{1}{h(1 / 2)(1+m)}\left(f\left(\frac{a+b}{2}\right)\left({ }_{g} F_{\mu, \alpha^{\prime}, l, a^{+}}^{\phi, \gamma, \delta, c} 1\right)(b, \omega ; p)+\left({ }_{g} F_{\mu, \beta, l, b^{-}}^{\phi, \gamma, \delta, k, c} 1\right)(a, \omega ; p)\right) \\
& +\lambda h\left(\frac{1}{2}\right) h\left(\frac{1}{2}\right)\left(\left(F_{\mu, \alpha^{\prime}, l, a^{+}}^{\phi, \gamma, \delta, k, c}(a+b-2 x)^{2}\right)(b, \omega ; p)\right. \\
& \left.+\left({ }_{g} F_{\mu, \beta, l, b^{-}}^{\phi, \gamma, \delta, k, c}(a+b-2 x)^{2}\right)(a, \omega ; p)\right) \leq\left({ }_{g} F_{\mu, \alpha^{\prime}, l, a^{+}}^{\phi, \gamma, \delta, k, c} f\right)(b, \omega ; p) \\
& \quad+\left({ }_{g} F_{\mu, \beta, l, b^{-}}^{\phi, \gamma, \delta, k} f\right)(a, \omega ; p) \leq(b-a)\left(K_{b}^{a}\left(E_{\mu, \alpha^{\prime}, l}^{\gamma, \delta, k, c}, g ; \phi\right)+K_{b}^{a}\left(E_{\mu, \beta, l}^{\gamma, \delta, k, c}, g ; \phi\right)\right) \\
& \left(f(b) Y_{b}^{a}\left(z, h ; g^{\prime}\right)+f(a) Y_{b}^{a}\left(y, h ; g^{\prime}\right)-\lambda(b-a) h(1)(g(b)-g(a))\right) .
\end{aligned}
$$


The following remark summarizes the connection of Theorem 8 with already known results.

\section{Remark 4}

(i) If we consider $\eta=0, \lambda=0, \alpha^{\prime}=\beta, m=\alpha=1$, and $h(t)=t$ in (35), then Theorem 22 of [38] can be obtained

(ii) If we consider $\eta=0, \lambda=0, \phi(t)=\Gamma\left(\alpha^{\prime}\right) t^{\left(\alpha^{\prime} / k\right)+1}$, $h(t)=g(t)=t, \alpha=m=1$, and $p=\omega=0$ in (35), then Theorem 3 of [13] can be obtained

(iii) If we consider $\eta=0, \lambda=0$, and $\alpha^{\prime}=\beta$ in the result of (ii), then Corollary 6 of [13] can be obtained

(iv) If we consider $\eta=0, \lambda=0, \phi(t)=\Gamma\left(\alpha^{\prime}\right) t^{\alpha^{\prime}+1}$, $p=\omega=0, m=\alpha=1$, and $g(t)=h(t)=t$ in (35), then Theorem 3 of [15] can be obtained

(vi) If we consider $\eta=0, \quad \lambda=0, \quad \alpha^{\prime}=\beta$, and $\phi(t)=\Gamma\left(\alpha^{\prime}\right) t^{\alpha^{\prime}}$ in the result of $(\mathrm{v})$, then Corollary 6 of [15] can be obtained (vii) If we consider $\eta=0, \lambda=0, \phi(t)=\Gamma\left(\alpha^{\prime}\right) t^{\alpha^{\prime}+1}$, $p=\omega=0, m=\alpha=1$, and $h(t)=t$ in (35), then Theorem 3 of [17] can be obtained

(viii) If we consider $\eta=0, \lambda=0$, and $\alpha^{\prime}=\beta$ in the result of (vii), then Corollary 3 of [17] can be obtained

(ix) If we consider $\eta=0, \lambda=0, \phi(t)=t^{\alpha^{\prime}+1}, g(t)=t$, $h(t)=t^{s}$, and $\alpha=m=1$ in (35), then Theorem 2.4 of [43] can be obtained

(x) If we consider $\eta=0, \lambda=0$, and $\alpha^{\prime}=\beta$ in the result of (ix), then Corollary 2.6 of [43] can be obtained

The forthcoming result is established for a differentiable function $f$ by utilizing the strongly exponentially $(\alpha, h-m)$-convexity of $\left|f^{\prime}\right|$.

Theorem 9. Let $f:[a, b] \longrightarrow \mathbb{R}$ be a differentiable function. $\left|f^{\prime}\right|$ is strongly exponentially $(\alpha, h-m)$-convex. Let $g:[a, b] \longrightarrow \mathbb{R}$ be a differentiable and strictly increasing function; also, let $(\phi / x)$ be an increasing function on $[a, b]$ and $h(x) h(y) \leq h(x+y)$. If $\alpha^{\prime}, \beta, l, \gamma, c \in \mathbb{R}_{+}, p, \mu \geq 0, \delta \geq 0$, and $0<k \leq \delta+\mu$, then for $x \in(a, b)$, we have

$$
\begin{aligned}
& \left|\left(g_{g, \alpha^{\prime}, l, a^{+}}^{\phi, \gamma, \delta, k}(f * g)\right)(x, \omega ; p)+\left(g_{\mu, \beta, l, b^{-}}^{\phi, \gamma, \delta, k, c}(f * g)\right)(x, \omega ; p)\right| \leq K_{x}^{a}\left(E_{\mu, \alpha^{\prime}, l}^{\gamma, \delta, k, c}, g ; \phi\right) \\
& \quad \times(x-a)\left(\frac{\left|f^{\prime}(a)\right|}{e^{\eta a}} X_{x}^{a}\left(z^{\alpha}, h ; g^{\prime}\right)+m \frac{\left|f^{\prime}(x / m)\right|}{e^{\eta(x / m)}} X_{x}^{a}\left(1-z^{\alpha}, h ; g^{\prime}\right)\right. \\
& \left.\quad-\frac{\lambda(x-m a)^{2} h(1)(g(x)-g(a))}{m(x-a)}\right)+K_{b}^{x}\left(E_{\mu, \beta, l}^{\gamma, \delta, k, c}, g ; \phi\right)(b-x) \\
& \quad \times\left(\frac{\left|f^{\prime}(b)\right|}{e^{\eta b}} X_{x}^{b}\left(z^{\alpha}, h ; g^{\prime}\right)+\frac{m\left|f^{\prime}(x / m)\right|}{e^{\eta(x / m)}} X_{x}^{b}\left(1-z^{\alpha}, h ; g^{\prime}\right)-\frac{\lambda(m b-x)^{2} h(1)(g(b)-g(x))}{m(b-x)}\right),
\end{aligned}
$$

where

$$
\begin{aligned}
& \left({ }_{g} F_{\mu, \alpha^{\prime}, l, a^{+}}^{\phi, \gamma, \delta, k, c} f * g\right)(x, \omega ; p):=\int_{a}^{x} K_{x}^{t}\left(E_{\mu, \alpha^{\prime}, l}^{\gamma, \delta, k, c}, g ; \phi\right) f^{\prime}(t) d(g(t)), \\
& \left({ }_{g} F_{\mu, \beta, l, b^{-}}^{\phi, \gamma, \delta, k, c} f * g\right)(x, \omega ; p):=\int_{x}^{b} K_{t}^{x}\left(E_{\mu, \alpha^{\prime}, l}^{\gamma, \delta, k, c}, g ; \phi\right) f^{\prime}(t) d(g(t)) .
\end{aligned}
$$

Proof. Using the strongly exponentially $(\alpha, h-m)$-convexity of $\left|f^{\prime}\right|$, we have

$$
\begin{aligned}
\left|f^{\prime}(t)\right| \leq & h\left(\frac{x-t}{x-a}\right)^{\alpha} \frac{\left|f^{\prime}(a)\right|}{e^{\eta a}}+m h\left(1-\left(\frac{x-t}{x-a}\right)^{\alpha}\right) \frac{\left|f^{\prime}(x / m)\right|}{e^{\eta(x / m)}} \\
& -\frac{\lambda(x-a m)^{2}}{m e^{\eta(a+(x / m))}} h\left(\frac{x-t}{x-a}\right)^{\alpha} h\left(1-\left(\frac{x-t}{x-a}\right)^{\alpha}\right),
\end{aligned}
$$

from which we can write the following inequality:

$$
\begin{aligned}
& -\left(h\left(\frac{x-t}{x-a}\right)^{\alpha} \frac{\left|f^{\prime}(a)\right|}{e^{\eta a}}+m h\left(1-\left(\frac{x-t}{x-a}\right)^{\alpha}\right) \frac{\left|f^{\prime}(x / m)\right|}{e^{\eta(x / m)}}\right. \\
& \left.-\frac{\lambda(x-a m)^{2}}{m e^{\eta(a+(x / m))}} h\left(\frac{x-t}{x-a}\right)^{\alpha} h\left(1-\left(\frac{x-t}{x-a}\right)^{\alpha}\right)\right) \leq f^{\prime}(t) \\
& \leq\left(h\left(\frac{x-t}{x-a}\right)^{\alpha} \frac{\left|f^{\prime}(a)\right|}{e^{\eta a}}+m h\left(1-\left(\frac{x-t}{x-a}\right)^{\alpha}\right) \frac{\left|f^{\prime}(x / m)\right|}{e^{\eta(x / m)}}\right. \\
& \left.-\frac{\lambda(x-a m)^{2}}{m e^{\eta(a+(x / m))}} h\left(\frac{x-t}{x-a}\right)^{\alpha} h\left(1-\left(\frac{x-t}{x-a}\right)^{\alpha}\right)\right) .
\end{aligned}
$$


Let us consider the other inequality of (51):

$$
\begin{array}{r}
f^{\prime}(t) \leq h\left(\frac{x-t}{x-a}\right)^{\alpha} \frac{\left|f^{\prime}(a)\right|}{e^{\eta a}}+m h\left(1-\left(\frac{x-t}{x-a}\right)^{\alpha}\right) \frac{\left|f^{\prime}(x / m)\right|}{e^{\eta(x / m)}} \\
-\frac{\lambda(x-a m)^{2}}{m e^{\eta(a+(x / m))}} h\left(\frac{x-t}{x-a}\right)^{\alpha} h\left(1-\left(\frac{x-t}{x-a}\right)^{\alpha}\right) .
\end{array}
$$

Multiplying (23) and (52) and integrating over $[a, x]$, we obtain

$$
\begin{aligned}
& \int_{a}^{x} K_{x}^{t}\left(E_{\mu, \alpha^{\prime}, l}^{\gamma, \delta, c, c}, g ; \phi\right) f^{\prime}(t) d(g(t)) \leq \frac{\left|f^{\prime}(a)\right|}{e^{\eta a}} K_{x}^{a}\left(E_{\mu, \alpha^{\prime}, l}^{\gamma, \delta, k, c}, g ; \phi\right) \\
& \quad \times \int_{a}^{x} h\left(\frac{x-t}{x-a}\right)^{\alpha} d(g(t))+m \frac{\left|f^{\prime}(x / m)\right|}{e^{\eta(x / m)}} K_{x}^{a}\left(E_{\mu, \alpha^{\prime}, l}^{\gamma, \delta, k, c}, g ; \phi\right) \\
& \quad \times \int_{a}^{x} h\left(1-\left(\frac{x-t}{x-a}\right)^{\alpha}\right) d(g(t))-\frac{\lambda(x-m a)^{2}}{m e^{\eta(a+(x / m))}} K_{x}^{a}\left(E_{\mu, \alpha^{\prime}, l}^{\gamma, \delta, k, c}, g ; \phi\right) \\
& \quad \times \int_{a}^{x} h\left(1-\left(\frac{x-t}{x-a}\right)^{\alpha}\right) h\left(\frac{x-t}{x-a}\right)^{\alpha} d(g(t)),
\end{aligned}
$$

from which we get

$$
\begin{aligned}
& \left({ }_{g} F_{\mu, \alpha^{\prime}, l, a^{+}}^{\phi, \gamma, \delta, k, c} f\right)(x, \omega ; p) \leq K_{x}^{a}\left(E_{\mu, \alpha^{\prime}, l}^{\gamma, \delta, k, c}, g ; \phi\right)(x-a) \\
& \quad \times\left(\frac{\left|f^{\prime}(a)\right|}{e^{\eta a}} X_{x}^{a}\left(z^{\alpha}, h ; g^{\prime}\right)+m \frac{\left|f^{\prime}(x / m)\right|}{e^{\eta(x / m)}} X_{x}^{a}\left(1-z^{\alpha}, h ; g^{\prime}\right)\right. \\
& \left.\quad-\frac{\lambda(x-m a)^{2} h(1)(g(x)-g(a))}{m(x-a) e^{\eta(a+(x / m))}}\right) .
\end{aligned}
$$

If we consider the first inequality of (51) and adopt the same pattern as we did for the second inequality, then we have

$$
\begin{aligned}
& \left({ }_{g} F_{\mu, \alpha^{\prime}, l, a^{+}}^{\phi, \gamma, \delta, c} g f\right)(x, \omega ; p) \geq-K_{x}^{a}\left(E_{\mu, \alpha^{\prime}, l}^{\gamma, \delta, c, c}, g ; \phi\right)(x-a) \\
& \quad \times\left(\frac{\left|f^{\prime}(a)\right|}{e^{\eta a}} X_{x}^{a}\left(z^{\alpha}, h ; g^{\prime}\right)+m \frac{\left|f^{\prime}(x / m)\right|}{e^{\eta(x / m)}} X_{x}^{a}\left(1-z^{\alpha}, h ; g^{\prime}\right)\right. \\
& \left.\quad-\frac{\lambda(x-m a)^{2} h(1)(g(x)-g(a))}{m(x-a) e^{\eta(a+(x / m))}}\right) .
\end{aligned}
$$

From (54) and (55), the following inequality is obtained:

$$
\begin{aligned}
& \left({ }_{g} F_{\mu, \alpha^{\prime}, l, a^{+}}^{\phi, \gamma, \delta, k, c} f\right)(x, \omega ; p) \mid \leq K_{x}^{a}\left(E_{\mu, \alpha^{\prime}, l}^{\gamma, \delta, k, c}, g ; \phi\right)(x-a) \\
& \quad \times\left(\frac{\left|f^{\prime}(a)\right|}{e^{\eta a}} X_{x}^{a}\left(z^{\alpha}, h ; g^{\prime}\right)+m \frac{\left|f^{\prime}(x / m)\right|}{e^{\eta(x / m)}} X_{x}^{a}\left(1-z^{\alpha}, h ; g^{\prime}\right)\right. \\
& \left.\quad-\frac{\lambda(x-m a)^{2} h(1)(g(x)-g(a))}{m(x-a) e^{\eta(a+(x / m))}}\right) .
\end{aligned}
$$

Now, using the strongly exponentially $(\alpha, h-m)$-convexity of $\left|f^{\prime}\right|$, we have

$$
\begin{aligned}
\left|f^{\prime}(t)\right| \leq & h\left(\frac{t-x}{b-x}\right)^{\alpha} \frac{\left|f^{\prime}(b)\right|}{e^{\eta b}}+m h\left(1-\left(\frac{t-x}{b-x}\right)^{\alpha}\right) \frac{\left|f^{\prime}(x / m)\right|}{e^{\eta(x / m)}} \\
& -\frac{\lambda(b m-x)^{2}}{m e^{\eta(b+(x / m))}} h\left(\frac{t-x}{b-x}\right)^{\alpha} h\left(1-\left(\frac{t-x}{b-x}\right)^{\alpha}\right) .
\end{aligned}
$$

On the same pattern as we did for (23) and (50), one can obtain the following inequality from (24) and (58):

$$
\begin{aligned}
& \left|\left({ }_{g} F_{\mu, \beta, l, b^{-}}^{\phi, \gamma, \delta, k} f\right)(x, \omega ; p)\right| \leq K_{b}^{x}\left(E_{\mu, \beta, l}^{\gamma, \delta, k, c}, g ; \phi\right)(b-x) \\
& \quad \times\left(\frac{\left|f^{\prime}(b)\right|}{e^{\eta b}} X_{x}^{b}\left(z^{\alpha}, h ; g^{\prime}\right)+m \frac{\left|f^{\prime}(x / m)\right|}{e^{\eta(x / m)}} X_{x}^{b}\left(1-z^{\alpha}, h ; g^{\prime}\right)\right. \\
& \left.\quad-\frac{\lambda(b m-x)^{2} h(1)(g(b)-g(x))}{m(b-x) e^{\eta(b+(x / m))}}\right) .
\end{aligned}
$$

By adding (56) and (58), inequality (48) can be achieved.

Corollary 3. If we take $\eta=0$ and $m=\alpha=1$, then (48) holds for strongly h-convex functions.

$$
\begin{aligned}
& \left({ }_{g} F_{\mu, \alpha^{\prime}, l, a^{+}}^{\phi, \gamma, \delta, c} f * g\right)(x, \omega ; p)+\left({ }_{g} F_{\mu, \beta, l, b^{-}}^{\phi, \gamma, \delta, k} f * g\right)(x, \omega ; p) \leq K_{x}^{a}\left(E_{\mu, \alpha^{\prime}, l}^{\gamma, \delta, k, c}, g ; \phi\right)(x-a) \\
& \left(\left|f^{\prime}(a)\right| Y_{x}^{a}\left(z, h ; g^{\prime}\right)+\left|f^{\prime}(x)\right| Y_{x}^{a}\left(y, h ; g^{\prime}\right)-\lambda(x-a) h(1)(g(x)-g(a))\right) \\
& \quad+K_{b}^{x}\left(E_{\mu, \beta, l}^{\gamma, \delta, k, c}, g ; \phi\right)(b-x)\left(\left|f^{\prime}(b)\right| Y_{x}^{b}\left(z, h ; g^{\prime}\right)+\left|f^{\prime}(x)\right| Y_{x}^{b}\left(y, h ; g^{\prime}\right)\right. \\
& \quad-\lambda(b-x) h(1)(g(b)-g(x))) .
\end{aligned}
$$


The following remark summarizes the connection of Theorem 9 with already known results.

\section{Remark 5}

(i) If we consider $\eta=0, \lambda=0$, and $\alpha=1$ in (48), then Theorem 3 of [40] can be obtained

(ii) If we consider $\eta=0, \lambda=0, \alpha^{\prime}=\beta$, and $h(t)=t$ in (48), then Theorem 3 of [14] can be obtained

(iii) If we consider $\eta=0, \lambda=0, \alpha^{\prime}=\beta, m=\alpha=1$, and $h(t)=t$ in (48), then Theorem 25 of [38] can be obtained

(iv) If we consider $\eta=0, \lambda=0, \phi(t)=\Gamma\left(\alpha^{\prime}\right) t^{\left(\alpha^{\prime} / k\right)+1}$, $h(t)=g(t)=t, \alpha=m=1$, and $p=\omega=0$ in (48), then Theorem 2 of [13] can be obtained

(v) If we consider $\eta=0, \lambda=0$, and $\alpha^{\prime}=\beta$ in the result of (iv), then Corollary 4 of [13] can be obtained

(vi) If we consider $\eta=0, \lambda=0, \phi(t)=t^{\alpha^{\prime}}, \alpha=1$, and $g(t)=t$ in (48), then Theorem 2 of [11] can be obtained

(vii) If we consider $\eta=0, \lambda=0, m=1$, and $h(t)=t$ in the result of (vi), then Corollary 2 of [11] can be obtained

(viii) If we consider $\eta=0, \lambda=0, \phi(t)=\Gamma\left(\alpha^{\prime}\right) t^{\alpha^{\prime}+1}$, $\alpha=1, \quad p=\omega=0$, and $g(t)=t$ in (48), then Theorem 2 of [12] can be obtained

(ix) If we consider $\eta=0, \lambda=0$, and $\alpha^{\prime}=\beta$ in the result of (viii), then Corollary 4 of [12] can be obtained

(x) If we consider $\eta=0, \lambda=0, \phi(t)=\Gamma\left(\alpha^{\prime}\right) t^{\alpha^{\prime}+1}$, $p=\omega=0, m=\alpha=1$, and $g(t)=h(t)=t$ in (48), then Theorem 2 of [15] can be obtained

(xi) If we consider $\eta=0, \lambda=0, \alpha^{\prime}=\beta$, and $\phi(t)=$ $\Gamma\left(\alpha^{\prime}\right) t^{\alpha^{\prime}}$ in the result of $(\mathrm{x})$, then Corollary 4 of [15] can be obtained

(xii) If we consider $\eta=0, \lambda=0, \phi(t)=\Gamma\left(\alpha^{\prime}\right) t^{\alpha^{\prime}+1}$, $p=\omega=0, m=\alpha=1$, and $h(t)=t$ in (48), then Theorem 2 of [17] can be obtained

(xiii) If we consider $\eta=0, \lambda=0$, and $\alpha^{\prime}=\beta$ in the result of (xii), then Corollary 2 of [17] can be obtained

(xiv) If we consider $\eta=0, \lambda=0, \phi(t)=t^{\alpha^{\prime}+1}, g(t)=t$, $h(t)=t^{s}$, and $\alpha=m=1$ in (48), then Theorem 2.3 of [43] can be obtained

(xv) If we consider $\eta=0, \lambda=0$, and $\alpha^{\prime}=\beta$ in the result of (xiv), then Corollary 2.5 of [43] can be obtained

(xvi) If we consider $\eta=0, \lambda=0, p=\omega=0, \alpha=1$, and $h(t)=t^{s}$ in (48), then Theorem 2 of [44] can be obtained

(xvii) If we consider $\eta=0, \lambda=0, \phi(t)=\Gamma\left(\alpha^{\prime}\right) t^{\alpha^{\prime}}$, $h(t)=t^{s}, \alpha=1$, and $g(t)=t$ in (48), then Theorem 3 of [41] can be obtained (xviii) If we consider $\eta=0, \lambda=0$, and $\alpha^{\prime}=\beta$ in the result of (xvii), then Corollary 5 of [41] can be obtained

(xix) If we consider $\eta=0, \lambda=0, \phi(t)=\Gamma\left(\alpha^{\prime}\right) t^{\left(\alpha^{\prime} / k\right)+1}$, $h(t)=t$, and $p=\omega=0$ in (48), then Theorem 2 of [21] can be obtained

(xx) If we consider $\eta=0, \lambda=0$, and $\alpha^{\prime}=\beta$ in the result of (xix), then Corollary (2) of [21] can be obtained

\section{Concluding Remarks}

Recently defined functions of different kinds related to the notion of convexity are combined in a single definition (Definition 9). From this definition, the reader can obtain at once convex, strongly convex, and exponentially convex functions of various kinds defined by different authors in near past decades in [3-6, 19, 42, 45-51]. Therefore, this paper provides new as well as results of several published papers in compact forms. The reader simultaneously can obtain inequalities for fractional integrals of many types for various kinds of convex functions by specific settings of involved functions and parameters. The remarks elaborate connections of the proved results with well-known inequalities that already exist in the literature.

\section{Data Availability}

No data were used to support this study.

\section{Conflicts of Interest}

The authors declare that they have no conflicts of interest.

\section{References}

[1] S. Abramovich, G. Jameson, and G. Sinnamon, "Refining Jensen's inequality," Bulletin mathématiques de la Société des sciences mathématiques de Roumanie, vol. 47, no. 1-2, pp. 3-14, 2004.

[2] D. A. Ion, "Some estimates on the Hermite-Hadamard inequality through quasi-convex functions," Annals of the University of Craiova-Mathematics and Computer Science Series, vol. 34, pp. 82-87, 2007.

[3] V. G. Mihesan, A Generalization of the Convexity, Seminar on Functional Equations, Approx and Convex, Open Access and Academic Publisher, Cluj-Napoca, Romania, 1993.

[4] M. E. Özdemir, A. O. Akdemri, and E. Set, "On $(h-m)$-convexity and Hadamard-type inequalities," Transylvanian Journal of Mathematics and Mechanics, vol. 8, no. 1, pp. 51-58, 2016.

[5] B. T. Polyak, "Existence theorems and convergence of minimizing sequences in extremum problems with restrictions," Soviet Doklady Mathematics, vol. 7, pp. 72-75, 1966.

[6] S. Varošanec, "On h-convexity," Journal of Mathematical Analysis and Applications, vol. 326, no. 1, pp. 303-311, 2007.

[7] M. Andrić, G. Farid, and J. Pečarić, "A further extension of Mittag-Leffler function," Fractional Calculus and Applied Analysis, vol. 21, no. 5, pp. 1377-1395, 2018. 
[8] P. Agarwal, "Some inequalities involving Hadamard-type k -fractional integral operators," Mathematical Methods in the Applied Sciences, vol. 40, no. 11, pp. 3882-3891, 2017.

[9] P. Agarwal, M. Jleli, and M. Tomar, "Certain HermiteHadamard type inequalities via generalized k-fractional integrals," Journal of Inequalities and Applications, vol. 2017, p. 55, 2017.

[10] H. Chen and U. N. Katugampola, "Hermite-Hadamard and Hermite-Hadamard-Fejér type inequalities for generalized fractional integrals," Journal of Mathematical Analysis and Applications, vol. 446, no. 2, pp. 1274-1291, 2017.

[11] Z. Chen, G. Farid, A. U. Rehman, and N. Latif, "Estimation of fractional integral operators via convex functions and related results," Advances in Difference Equations, vol. 2020, no. 163, 2020.

[12] G. Farid, "Bounds of Riemann-Liouville fractional integral operators," Computational Methods for Differential Equations, vol. 9, no. 2, pp. 637-648, 2021.

[13] G. Farid, "Estimations of Riemann-Liouville k-fractional integrals via convex functions," Acta et Commentationes Universitatis Tartuensis de Mathematica, vol. 23, no. 1, pp. 71-78, 2019.

[14] G. Farid, B. Ni, and K. Mahreen, "Inequalities for a unified integral operator via $(\alpha, m)$-convex functions," Jurnal Matematika, vol. 2020, Article ID 2345416, 9 pages, 2020.

[15] G. Farid, "Some Riemann-Liouville fractional integral inequalities for convex functions," Journal of Analytical, vol. 27, 2018.

[16] G. Farid, "A unified integral operator and further its consequences," Open Journal of Mathematical Analysis, vol. 4, no. 1, pp. 1-7, 2020.

[17] G. Farid, W. Nazeer, M. Saleem, S. Mehmood, and S. Kang, "Bounds of Riemann-Liouville fractional integrals in general form via convex functions and their applications," Mathematics, vol. 6, no. 11, p. 248, 2018.

[18] G. Farid, "Existence of an integral operator and its consequences in fractional and conformable integrals," Open Journal of Mathematical Sciences, vol. 3, no. 3, pp. 210-216, 2019.

[19] G. Farid, A. U. Rehman, and Q. U. Ain, " $k$-fractional integral inequalities of Hadamard type for $(h-m)$-convex functions," Computational Methods for Differential Equations, vol. 7, no. 5, pp. 1-22, 2019.

[20] S. Habib, S. Mubeen, and M. N. Naeem, "Chebyshev type integral inequalities for generalized $k$-fractional conformable integrals," Journal of Inequalities and Special Functions, vol. 9, no. 4, pp. 53-65, 2018.

[21] S. M. Kang, G. Farid, M. Waseem, S. Ullah, W. Nazeer, and S. Mehmood, "Generalized k-fractional integral inequalities associated with (alpha, $m$ )-convex functions," Journal of Inequalities and Applications, vol. 2019, no. 1, p. 255, 2019.

[22] Y. C. Kwun, G. Farid, W. Nazeer, S. Ullah, and S. M. Kang, "Generalized riemann-liouville k-fractional integrals associated with Ostrowski type inequalities and error bounds of hadamard inequalities," IEEE Access, vol. 6, pp. 64946-64953, 2018.

[23] M. Tomar, P. Agarwal, and J. Choi, "Hermite-Hadamard type Inequalies for generalized convex functions on fractal sets style," Boletim da Sociedade Paranaence de Mathematica, vol. 38, no. 1, pp. 101-116, 2020.

[24] H. J. Houbold, A. M. Mathai, and R. K. Saxena, "Mittag-Leffler functions and their applications," Journal of Applied Mathematics, vol. 2011, Article ID 298628, 51 pages, 2011.
[25] T. R. Parbhakar, "A singular integral equation with a generalized Mittag-Leffler function in the kernel," The Yokohama Mathematical Journal, vol. 19, pp. 7-15, 1971.

[26] Z. Chen, G. Farid, M. Saddiqa, S. Ullah, and N. Latif, "Study of fractional integral inequalities involving Mittag-Leffler functions via convexity," Journal of Inequalities and Applications, vol. 2020, no. 206, 2020.

[27] Z. He, X. Ma, X. Ma, G. Ul Haq, and K. Mahreen, "Bounds of a unified integral operator for $(s, m)$-convex functions and their consequences," AIMS Mathematics, vol. 5, no. 6, pp. 5510-5520, 2020.

[28] S. S. Dragomir, “Inequalities of Jensen's type for generalized $\mathrm{k}$-g-fractional integrals of function $\$ \mathrm{f} \$$ for which the composite $\mathrm{f} \bigcirc \mathrm{g}^{-1}$ is convex," Fractional Differential Calculus, vol. 8, no. 1, pp. 127-150, 2018.

[29] F. Jarad, E. Uğurlu, T. Abdeljawad, and D. Baleanu, "On a new class of fractional operators," Advances in Difference Equations, vol. 2017, no. 1, p. 247, 2017.

[30] T. U. Khan and M. A. Khan, "Generalized conformable fractional operators," Journal of Computational and Applied Mathematics, vol. 346, pp. 378-389, 2019.

[31] A. A. Kilbas, H. M. Srivastava, and J. J. Trujillo, "Theory and applications of fractional differential equations," North-Holland Mathematics Studies, Elsevier, New York, NY, USA, 2006.

[32] S. Mubeen and G. M. Habibullah, " $k$-fractional integrals and applications," International Journal of Contemporary Mathematical Sciences, vol. 7, no. 2, pp. 89-94, 2012.

[33] G. Rahman, D. Baleanu, M. A. Qurashi, S. D. Purohit, S. Mubeen, and M. Arshad, "The extended Mittag-Leffler function via fractional calculus," The Journal of Nonlinear Science and Applications, vol. 10, pp. 4244-4253, 2013.

[34] T. O. Salim and A. W. Faraj, "A generalization of MittagLeffler function and integral operator associated with integral calculus," Fractional Calculus and Applied Analysis, vol. 3, no. 5, pp. 1-13, 2012.

[35] M. Z. Sarikaya, Z. Dahmani, M. E. Kiris, and F. Ahmad, "( $k$, s)-Riemann-Liouville fractional integral and applications," Hacettepe Journal of Mathematics and Statistics, vol. 1, no. 45, pp. 77-89, 2016.

[36] H. M. Srivastava and Ž. Tomovski, "Fractional calculus with an integral operator containing a generalized Mittag-Leffler function in the kernel," Applied Mathematics and Computation, vol. 211, no. 1, pp. 198-210, 2009.

[37] T. Tunc, H. Budak, F. Usta, and M. Z. Sarikaya, "On new generalized fractional integral operators and related fractional inequalities," 2017, https://www.researchgate.net/publication/ 313650587.

[38] Y. C. Kwun, G. Farid, S. Ullah, W. Nazeer, K. Mahreen, and S. M. Kang, "Inequalities for a unified integral operator and associated results in fractional calculus," IEEE Access, vol. 7, pp. 126283-126292, 2019.

[39] C. Y. Jung, G. Farid, M. Andrić, J. Pečarić, and Y. M. Chu, "Refinements of some integral inequalities for unified integral operators," Journal of Inequalities and Applications, vol. 2021, p. 7, 2021.

[40] G. Farid and K. Mahreen, "Study of inequalities for unified integral operators of generalized convex functions," Open Journal of Mathematical Analysis, vol. 5, 2021.

[41] G. Farid, S. B. Akbar, S. Ur Rehman, and J. Pečarić, "Boundedness of fractional integral operators containing Mittag-Leffler functions via $(s, m)$-convexity," AIMS Mathematics, vol. 5, no. 2, pp. 966-978, 2020. 
[42] M. Bracamonte, J. Giménez, and M. Vivas-Cortez, "Hermitehadamard-fejer type inequalities for strongly $(s, m)$-Convex functions with modulus c, in second sense," Applied Mathematics \& Information Sciences, vol. 10, no. 6, pp. 2045-2053, 2016.

[43] L. Chen, G. Farid, S. I. But, and S. B. Akbar, "Boundedness of fractional integral operators containing Mittag-Leffler functions," Turkish Journal of Inequalities, vol. 4, no. 1, pp. 14-24, 2020.

[44] Y. C. Kwun, G. Farid, S. M. Kang, B. K. Bangash, and S. Ullah, "Derivation of bounds of several kinds of operators via $(s, m)$-convexity," Advances in Difference Equations, vol. 2020 , no. 5,2020 .

[45] G. A. Anastassiou, "Generalized fractional Hermite Hadamard inequalities involving $m$-convexity and $(s, m)$-convexity," Series: Mathematics and Informatics, vol. 28, no. 2, pp. 107-126, 2013.

[46] M. U. Awan, M. A. Noor, and K. I. Noor, "Hermite-Hadamard inequalities for exponentially convex functions," Applied Mathematics \& Information Sciences, vol. 12, no. 2, pp. 405-409, 2018.

[47] S. S. Dragomir, "Integral inequalities of Jensen type for $\lambda$-convex functions," Matematiqki Vesnik, vol. 68, no. 1 , pp. 45-57, 2016.

[48] E. K. Godunova and V. I. Levin, "Inequalities for functions of a broad class that contains convex, monotone and some other forms of functions," Numerical Mathematical physics, pp. 138-142, 1985, in Russian.

[49] N. Mehreen and M. Anwar, "Hermite-Hadamard type inequalities for exponentially $\mathrm{p}$-convex functions and exponentially s-convex functions in the second sense with applications," Journal of Inequalities and Applications, vol. 2019, no. 1, p. 92, 2019.

[50] X. Qiang, G. Farid, J. Pečarić, and S. B. Akbar, "Generalized fractional integral inequalities for exponentially $(s, m)$-convex functions," Journal of Inequalities and Applications, vol. 202070 pages, 2020.

[51] G. Toader, "Some generalizations of the convexity," Proceedings of the Colloquium on Approximation and Optimization, pp. 329-338, University of Cluj-Napoca, ClujNapoca, Romania, 1984. 